\title{
BMJ Global Health How and why front-line health workers (did not) use a multifaceted mHealth intervention to support maternal and neonatal healthcare decision-making in Ghana
}

\author{
Hannah Brown Amoakoh, ${ }^{1,2}$ Kerstin Klipstein-Grobusch, ${ }^{2,3}$ Evelyn Korkor Ansah, ${ }^{4}$ \\ Diederick E Grobbee, ${ }^{2}$ Linda Yveoo, ${ }^{5}$ Irene Agyepong ${ }^{5}$
}

To cite: Amoakoh HB, Klipstein-Grobusch K, Ansah EK, et al. How and why front-line health workers (did not) use a multifaceted mHealth intervention to support maternal and neonatal healthcare decision-making in Ghana. BMJ Glob Health 2019;4:e001153. doi:10.1136/ bmjgh-2018-001153

Handling editor Stephanie M Topp

- Additional material is published online only. To view please visit the journal online (http://dx.doi.org/10.1136/ bmjgh-2018-001153).

Received 3 September 2018 Revised 5 December 2018 Accepted 7 December 2018

Check for updates

C Author(s) (or their employer(s)) 2019. Re-use permitted under CC BY-NC. No commercial re-use. See rights and permissions. Published by BMJ.

For numbered affiliations see end of article.

Correspondence to Dr Hannah Brown Amoakoh; ansomaame@hotmail.com
ABSTRACT
Introduction Despite increasing use of mHealth interventions, there remains limited documentation of 'how and why' they are used and therefore the explanatory mechanisms behind observed effects on beneficiary health outcomes. We explored 'how and why' an mHealth intervention to support clinical decision-making by frontline providers of maternal and neonatal healthcare services in a low-resource setting was used. The intervention consisted of phone calls (voice calls), text messaging (short messaging service (SMS)), internet access (data) and access to emergency obstetric and neonatal protocols via an Unstructured Supplementary Service Data (USSD). It was delivered through individual-use and shared facility mobile phones with unique Subscriber Identification Module (SIM) cards networked in a Closed User Group. Methods A single case study with multiple embedded subunits of analysis within the context of a cluster randomised controlled trial of the impact of the intervention on neonatal health outcomes in the Eastern Region of Ghana was performed. We quantitatively analysed SIM card activity data for patterns of voice calls, SMS, data and USSD. We conducted key informant interviews and focus group discussions with intervention users and manually analysed the data for themes.

Results Overall, the phones were predominantly used for voice calls $(64 \%)$, followed by data $(28 \%)$, SMS $(5 \%)$ and USSD $(2 \%)$, respectively. Over time, use of all intervention components declined. Qualitative analysis showed that individual health worker factors (demographics, personal and work-related needs, perceived timeliness of intervention, tacit knowledge), organisational factors (resource availability, information flow, availability, phone ownership), technological factors (attrition of phones, network quality) and client perception of health worker intervention usage explain the pattern of intervention use observed.

Conclusion How and why the mHealth intervention was used (or not) went beyond the technology itself and was influenced by individual and context-specific factors. These must be taken into account in designing similar interventions to optimise effectiveness.

\section{Key questions}

What is already known?

- Mobile technology (mHealth) has the potential to improve health worker efficiency and health service utilisation particularly in low-resource settings.

What are the new findings?

- We observed a preferential use of project phones for making phone calls and then accessing the internet.

- Shared use project phones were sometimes unavailable to some members of the group sharing the phone, creating unequal access to the intervention; whereas individual-use phones were available unless they got lost or stolen.

What do the new findings imply?

- Clinical decision-making support mHealth interventions that are based on voice calls and make use of the internet to access treatment protocols and advice are more likely to be preferred by users in settings similar to the study context.

- Designing clinical decision-making mHealth support interventions for use on personal phones of frontline health workers could potentially ensure that al health workers have equal access at all times.

\section{INTRODUCTION}

\section{Background}

The use of mobile technology (mHealth)based interventions to support delivery of healthcare services has become increasingly popular in low-resource settings where it is envisaged as a tool to improve health worker efficiency and health service utilisation. ${ }^{1-3}$ mHealth has been applied in diverse areas such as the care of people living with HIV/ AIDS, maternal and child health, tuberculosis management, vaccination programmes, data collection, provider-to-provider 
communication, provider-to-client communication and clinical decision-making support. ${ }^{24-8}$

While there is some literature about how mHealth interventions were used, ${ }^{910}$ information regarding why these patterns of use were observed is scarce. mHealth interventions are expensive to start up ${ }^{11}$ and expensive to maintain. Their potential to bridge the gap in the provision of quality healthcare services in low-resource settings, however, makes them appealing. Despite the attractiveness of mHealth interventions, evaluations of their effectiveness on health outcomes have shown mixed results. ${ }^{12}$ Where mHealth interventions made positive impact on health outcomes, gains observed were only marginal. ${ }^{13-15}$ To increase the effectiveness of mHealth interventions on health outcomes requires improvement in their design. Knowledge of 'how and why' mHealth interventions are used (or not) to produce their observed effects can inform the much needed design improvements.

In Ghana, a lower-middle-income country, maternal and neonatal mortality rates (319 per 100000 live births ${ }^{16}$ and 25 deaths per 1000 live births, ${ }^{17}$ respectively) are still unacceptably high despite recent improvement. Following initial formative work in the Greater Accra Region to understand front-line health worker (FHW) decision-making for mothers and newborns, ${ }^{18}$ the Accelerate Project developed an mHealth intervention whose components were based on suggestions for clinical decision-making support by respondents in the formative study. ${ }^{18}$ The mHealth intervention thus aimed to support improved clinical decision-making among FHWs to further reduce maternal and neonatal mortality. ${ }^{190}$ After piloting and finalising the development of the intervention in the Greater Accra Region of Ghana with the active engagement of FHWs in that region, it was evaluated for its effects on neonatal mortality in a cluster randomised controlled trial (CRCT) in the neighbouring Eastern Region of Ghana.

\section{Description of the intervention}

The mHealth intervention consisted of four components-phone calls, text messaging, access to the internet and access to an Unstructured Supplementary Service Data (USSD) - that provided emergency protocols in response to selection from a short code drop-down menu. USSD is a communications protocol that allows two-way exchange of data between phone users and information linked to the predesigned short codes stored on a remote computer of a telecommunications company. This makes USSD more interactive than text messaging. Each response message linked to a short code is limited to a length of 150-182 alphanumeric characters. The messages in this intervention were created by the design team of FHWs, family physicians, obstetricians and paediatricians in the Greater Accra Region, drawing on the Ghana Safe Motherhood protocols. All four components of the intervention were part of a single composite intervention delivered on a non-smart mobile phone (table 1). Health workers were expected to use the phones primarily to access neonatal and maternal health emergency protocols via the USSD and obtain additional support from colleagues and the internet via the other intervention components. Each project mobile phone had a unique Subscriber Identification Module (SIM) card. All the SIM cards were networked in a Closed User Group (CUG) that allowed free and unlimited access to the USSD and voice calls between all the unique SIM cards registered to the intervention. In this regard, all intervention users were members of the CUG. Free credit on the phones also allowed calling and text messaging numbers outside the CUG; thus FHWs could use the phones for personal purposes. Monthly reminders regarding the availability of the USSD protocols were sent via text messaging to FHWs.

Each midwife at post in each health facility during the CRCT baseline assessment was provided with one mobile phone for their personal use labelled 'individual-use' phones. The CRCT baseline assessment showed that overall $30 \%$ of health facilities in the intervention arm (ie, all intervention districts combined) already had a shared functional work phone (range was $13 \%-55 \%$ by district). The project provided one mobile phone as a shared facility phone for all cadres of front-line providers of maternal and neonatal healthcare services in all facilities in the intervention districts. These shared facility phones were often received by the head of the facility's maternal unit or a community health officer (CHO) on behalf of the health facility. All mobile phones were distributed during training sessions organised by the researchers prior to the start of the intervention. During the training sessions, FHWs were taught how to use the intervention. FHWs were assumed to be familiar with the basic functioning of a mobile phone (making calls, texting and accessing the internet) so the trainings focused on how to use the USSD.

Each of the eight local government districts that formed the intervention clusters (table 2) had at least one district hospital and varying mix of health centres (HCs) and Community Health Planning and Services (CHPS) facilities. In all, 312 mobile phones were distributed to the eight intervention clusters that participated in the CRCT. Seventy-four were shared-use phones and the rest (238) were individual-use phones. Five of the individual-use phones (including their SIM cards) could not be traced back to the FHWs who received them at the start of the intervention as the users did not sign for them and efforts to reach these SIM card numbers were futile. These five SIM cards could also not be traced on the Vodafone database as they never logged unto the Vodafone server. A total of 307 SIM cards could thus be traced back to the facilities and FHWs who received them. Overall, hospitals, HCs and CHPS (and maternity homes) received 190, 66 and 51 SIM cards, respectively. Three extra SIM cards were assigned to the research team to facilitate communication with the FHWs and were excluded in analysis. 
Table 1 Components of the intervention

\begin{tabular}{|c|c|}
\hline Intervention component & Description \\
\hline Cell phones & $\begin{array}{l}\text { Distribution of the non-smart mobile phones by the research team to health facilities in the } \\
\text { intervention clusters (districts) either as a shared-use phone or as individual-use phone. Each } \\
\text { midwife was provided an individual-use phone and each health facility had a shared-use } \\
\text { phone. }\end{array}$ \\
\hline CUG & $\begin{array}{l}\text { A network of SIM cards with unlimited access to make free phone calls to other SIM cards } \\
\text { within the network. All intervention users constituted membership of the CUG. }\end{array}$ \\
\hline Text messaging & $\begin{array}{l}\text { Sending of up to } 100 \text { free short messaging service per month to SIM cards in as well as } \\
\text { outside the CUG. }\end{array}$ \\
\hline Data bundle & System that provides up to $25 \mathrm{MB}$ of free data per month to the project SIM cards. \\
\hline Monthly credit top-up & $\begin{array}{l}\text { *An automated system from the telecommunication company that topped up } 2.50 \text { cedis } \\
\text { (US } \$ 0.70) \text { worth of Vodafone credit on project SIM cards each month. This top up credit could } \\
\text { be used at the discretion of the health worker for making calls, texting or browsing the internet } \\
\text { beyond the limits set for text messaging and data bundle aforementioned. }\end{array}$ \\
\hline Reminders & $\begin{array}{l}\text { Monthly reminders sent to the intervention users reminding them of the availability of the USSD } \\
\text { protocols. }\end{array}$ \\
\hline Training & $\begin{array}{l}\text { Health workers were trained on how to use the intervention first at a group gathering in each } \\
\text { intervention district capital before the start of the cluster randomised controlled trial and then } \\
\text { at least once during monitoring visits in their individual health facilities during intervention } \\
\text { implementation. }\end{array}$ \\
\hline USSD & $\begin{array}{l}\text { A communications protocol that allows a two-way exchange of data between a phone } \\
\text { user and preprogramed information linked to short codes stored on a remote computer of } \\
\text { a telecommunication company. This makes it more interactive than text messaging. Each } \\
\text { response message linked to a short code is limited to a length of } 150-182 \text { alphanumeric } \\
\text { characters. In the intervention districts, it was used for requesting and receiving text-message- } \\
\text { based standard emergency obstetric and neonatal protocols on the request of a health } \\
\text { worker. Access to the USSD was limited to only project SIM cards (CUG members). For CUG } \\
\text { members, access to the USSD was free and with no limits to the number of times the USSD } \\
\text { could be accessed. }\end{array}$ \\
\hline
\end{tabular}

${ }^{*}$ Exchange rate of US $\$ 1=3.56$ cedis is based on the Bank of Ghana exchange rate at start of the intervention in August 2015.

CUG, Closed User Group; SIM, Subscriber Identification Module; USSD, Unstructured Supplementary Service Data.

Table 2 Background characteristics of clusters (districts) in the Eastern Region of Ghana at intervention baseline

\begin{tabular}{|c|c|c|c|c|c|c|c|c|c|c|}
\hline \multirow[b]{2}{*}{ District (cluster) } & \multicolumn{4}{|c|}{ Health facilities, n } & \multicolumn{2}{|c|}{$\begin{array}{l}\text { Demographic } \\
\text { distribution of } \\
\text { health facilities }\end{array}$} & \multirow[t]{2}{*}{$\begin{array}{l}\text { Midwives, } \\
\mathrm{n}\end{array}$} & \multirow[t]{2}{*}{$\begin{array}{l}\text { Doctors, } \\
n^{\star}\end{array}$} & \multirow[t]{2}{*}{$\begin{array}{l}\text { Deliveries } \\
\text { per } \\
\text { midwife, n }\end{array}$} & \multirow[t]{2}{*}{$\begin{array}{l}\text { Phones } \\
\text { received, } \\
\text { n† }\end{array}$} \\
\hline & Hospitalł & $\begin{array}{l}\text { Health } \\
\text { centres }\end{array}$ & CHPS & $\begin{array}{l}\text { Maternity } \\
\text { home§ }\end{array}$ & $\begin{array}{l}\text { Remote } \\
\text { (n, \%) }\end{array}$ & $\begin{array}{l}\text { Non- } \\
\text { remote } \\
(n, \%)\end{array}$ & & & & \\
\hline Afram Plains North & 1 & 1 & 7 & 0 & $7(77.8)$ & 2 (22.2) & 14 & 1 & 80.0 & 21 \\
\hline Denkyembour & 2 & 3 & 3 & 0 & $5(62.5)$ & $3(37.5)$ & 33 & - & 94.8 & 41 \\
\hline Kwaebibiraem & 1 & 3 & 2 & 0 & $3(50.0)$ & $3(50.0)$ & 19 & 2 & 107.6 & 25 \\
\hline Kwahu West & 1 & 8 & 1 & 1 & $4(36.4)$ & 7 (63.6) & 38 & 9 & 101.6 & 49 \\
\hline Lower Manya Krobo & 3 & 2 & 0 & 1 & $1(16.7)$ & 5 (83.3) & 47 & 10 & 75.0 & 54 \\
\hline
\end{tabular}

*Number of doctors from main district hospital not provided by hospital management in the case of Denkyembour district.

†This may differ slightly from the sum of the number of midwives and the number of health facilities in the cluster as some individualuse phone could not be traced as in the case of Afram Plains North and West Akim districts.

łlncludes both private and public hospitals.

§Includes only private maternity homes. 


\section{Research question}

Despite several documentations of use of mHealth interventions and some evaluations, very few studies document 'how and why' these interventions were used (or not). ${ }^{9}{ }^{10}$ Previous multifaceted mHealth interventions have not included as many options for accessing clinical decision-making support ${ }^{21-23}$ as the Accelerate Project's mHealth intervention. In this study, we asked the question 'how and why' was the Accelerate mHealth intervention used (or not)? Our specific objective was to describe patterns of use of the different components of the mHealth intervention by FHWs and explore the reasons for the observed patterns of use.

\section{Study context}

Ghana is a lower-middle-income country with a population of about 28 million people. Her gross national income per capita in 2016 was estimated at US $\$ 1390.00^{24}$ and her per capita health expenditure in 2015 was US\$79.59 ${ }^{25}$ Like many low-income and middle-income countries (LMICs), Ghana faces constraints with her health workforce. Her estimated number of doctors and nurses/midwives per 1000 people in 2010 was 0.096 and 0.926 , respectively. ${ }^{24}$ The country is further characterised by poor maternal and neonatal health indices which have improved over time but still fell short of the Millennium Development Goals (MDGs). During the era of the MDGs (1990-2015), Ghana's maternal mortality ratio steadily declined from 634 per 100000 live births to 319 per 100000 live births. ${ }^{26}$ Neonatal mortality, however, declined marginally from 30 per 1000 live births ${ }^{172}$ to 25 per $1000^{17}$ live births from 1999 to 2017.

The study region, the Eastern Region, lies in the south of Ghana and its population is approximately $10.7 \%$ of the total national population, ${ }^{28}$ making it the third most populous region in Ghana. The Eastern Region ranks sixth in terms of high neonatal mortality rate in Ghana $^{29}$ and stands to benefit from interventions aimed at improving neonatal healthcare. Table 2 summarises meso-context (health facilities, human resources, workload, ie, deliveries per midwife) of the eight intervention districts.

\section{METHODS}

\section{Study design}

This study design was an exploratory and explanatory single case study with multiple embedded units of analysis. The case was defined as "how and why a mobile phone-based front-line health worker clinical decision-making support intervention was used (or not)'. Each embedded subunit of analysis was defined as 'a district in which the intervention was deployed'. This case study was conducted within the broader context of a CRCT of the impact of the intervention on neonatal health outcomes in 16 districts in the Eastern Region of Ghana randomised into eight intervention and eight control districts (clusters). Each of the eight intervention districts was treated as an embedded subunit of analysis of the case study. The CRCT has been described in detail elsewhere.$^{20}$ A cluster in the CRCT and in this study is a district. Ghana is divided into 10 regions, each of which is further divided into geographic local government administrative areas known as districts.

\section{Data collection methods and sampling}

We used mixed quantitative and qualitative methods of data collection in each of the eight districts. Data sources included routine Vodafone call log data, key informant interviews (KIIs) with FHWs and facility managers, and focus group discussions (FGDs) with FHWs.

\section{Vodafone call log data}

The call log data were routinely collected by Vodafone Ghana, the telecommunication company that provided technical support for the intervention throughout the CRCT. We analysed all mobile call detail record subtypes (mCDRs) as logged on the Vodafone archived database regarding utilisation of the project's SIM cards for any purpose (phone calls, texting, accessing the USSD or use of data) during the first eight months of an 18-month intervention period. Data regarding CUG communication was included in this archived data. Prior to the data extraction, phone numbers assigned to the various users were collated such that each intervention user (FHW), the health facility as well as the cluster the user worked in was documented and coded in the Vodafone database. This ensured that SIM cards and phone numbers could be traced back to the cluster, health facility and FHW using the project phones.

\section{Focus group discussions and key informant interviews}

FGDs and KIIs aimed to provide explanatory insights into the patterns of use of the phones observed from analysing the call $\log$ data. We initially thought that perspectives and experiences of facility nurse managers might be different from those of front-line midwives. Since there were usually only one or two facility nurse managers to several front-line midwives, we planned to hold KIIs with the facility nurse managers and FGDs with the front-line midwives. The FGDs were to stimulate frank discussions of experiences and opinion about the intervention, while KIIs were used to obtain insight on how and why the intervention was used from a managerial view and shared-phone user's experience. No theories regarding the observed pattern of use of the intervention were postulated prior to qualitative interviews.

We conducted the qualitative interviews immediately after the CRCT closed to avoid introducing a confounding element into the intervention. We considered it important to reflect the three levels of healthcare delivery at district level in Ghana, that is, hospitals, HCs and CHPS compounds and zones and the differences between them. We therefore aimed to purposively select a facility from each of the three levels in each of the eight districts. Within each of the three levels in a given district, 
there was no clear indication of differences that required purposive selection. We therefore randomly selected one health facility from the several at each level within each of the eight districts to participate in KII, and two health facilities from each level to select respondents to participate in FGD. We sampled health facilities for KIIs and FGDs using a random sequence generator in Microsoft Excel $^{30}$ and sampled health facilities for KIIs first. After health facility selection for KIIs, the head of the maternity unit and the holder of a shared-use phone in hospitals and HCs were purposively sampled to be interviewed. In the CHPS compound, only the head of the maternity unit was interviewed as typically each CHPS compound had only one-shared use phone allocated to them by the project team.

Regarding FGDs, health facilities already selected for KIIs were excluded from the sample except where there were very few health facilities in a cluster. To ensure representation of health facilities from all levels of the healthcare system in FGDs, where there was one hospital in a cluster, the hospital was purposively selected to participate in FGDs. In instances where the same health facility was selected for both FGD and KII, the respondents for FGDs and KIIs were different. Following sampling of health facilities for FGDs, any FHW or midwife who had knowledge about the use of the project mobile phone was invited to participate in FGDs; the decision as to who exactly would attend the FGDs was made by the head of the health facility sampled.

At least one FGD and two KIIs were scheduled to be conducted in each of the eight districts (clusters) at a location arranged by the district health management team. The arranged venues were usually the district health administration office or hospital conference rooms in the cluster. We collected all qualitative data from 9 April 2018 until 27 April 2018. Each FGD consisted of FHWs from the different health facilities sampled within the district. We aimed to keep conducting FGDs and KIIs until no new themes were emerging (saturation). We estimated that this would mean about $4-8$ FGDs $^{31}$ and $6-10$ KIIs. We analysed the data from each interview immediately after it closed to inform whether to keep going or not. By the time we had completed data collection and analysis of one FGD in each of the eight districts, we realised we were finding the same themes in the analysis. We therefore stopped the FGDs. In the case of the KIIs, by the time we had completed nine KIIs in three districts we realised the themes were the same across the KIIs, across the districts and between the KIIs and the FGDs. We therefore stopped the KIIs and invited planned KII respondents in the remaining five districts to join their district FGD. Three of the investigators (HBA, LY and IAA) worked with one research assistant to collect the qualitative data. All FGDs and KIIs were conducted face-to-face and audiotaped to facilitate transcription of data collected with notes being taken by HBA as well. All KIIs and FGDs were conducted and transcribed in English. The KIIs lasted on average 28 min, while FGDs lasted averagely 1 hour 26 min.

\section{Data analysis}

Qualitative data analysis was done on a rolling basis after each FGD or KII. All data analysis was initially done by each of the eight districts for themes, commonalities and contrasts. The data were then compared across the eight districts for commonalities and contrasts. We triangulated the quantitative findings from the Vodafone call log data analysis and the qualitative findings from the FGD and KII.

\section{Vodafone call log}

Data were checked for errors and exported from Excel spreadsheets ${ }^{30}$ to Stata V.13 ${ }^{32}$ for cleaning and analysis. The category and the number of staff in maternity homes and CHPS are similar. Both facility types usually have 1-2 midwives who run the health facility post assisted by 2-3 CHOs to provide antenatal, neonatal and conduct routine normal uncomplicated deliveries. Some CHPS may however not have a midwife at post; in such situations, deliveries are only conducted if a pregnant woman presents in second stage of labour with the head of the baby in the perineum. In the case of some maternity homes, trained traditional birth attendants who work under the supervision of a midwife may be present. Due to the similarities in organisational structure, personnel and health services provided by CHPS and maternity homes participating in this study, the call log data from these two facility types were combined for analysis. We further classified health facilities into two groups of remote and non-remote areas based on access. Remote facilities were either located $>30$ min walk, or $>15$ min motorbike ride from the main district township, and had poor road access (uneven and untarred roads overcrowded with weeds and shrubs) leading to them. Non-remote health facilities were either located within $30 \mathrm{~min}$ walk or 15 min motorbike ride from the main district township, and had good road access leading to them. mCDRs for all explanatory variables of interest (clusters, level and location of health facility, type-of-phone (individual-use or shared-use)) were analysed and expressed in numbers and percentages. Analyses of the mCDRs were performed for the combined 8-month data and also disaggregated into monthly intervals for each explanatory variable. $\chi^{2}$ tests were applied to these analyses to assess the significance of the observed pattern of intervention usage. The SIM cards that used these mCDRs were analysed and expressed as percentages. Descriptive analysis of the CUG communication within and across each category of explanatory variable was also performed and expressed as number of voice and short messaging service (SMS) mCDRs records and their percentages. $\chi^{2}$ and Fisher's exact tests were applied to these analyses to assess the significance of the pattern of CUG communication. To further understand how the CUG communication was used in each cluster, we identified SIM cards that used the CUG and the health facilities they communicated with. No tests were applied to this in-depth analysis as there were several empty cells. 
Focus group discussions and key informant interviews

The voice recordings were transcribed during and continued after data collection. Transcriptions were done verbatim by non-data collectors. Each transcription was cross-checked by two persons (data collectors, including HBA). Data were manually analysed by thoroughly reading each transcript to identify themes, commonalities and contrasts emerging from the data that shed insights into the patterns of use of the intervention observed from the Vodafone call log data and why and how these patterns occurred using an inductive approach. Three of the study investigators performed the data analysis. Consensus on emerging themes was reached if a minimum of two of the data analyst agreed on an emerging theme.

\section{RESULTS}

In aggregate, $94 \%$ of the 307 SIM cards ever accessed the intervention during the first eight months of intervention implementation. Of the 307 SIM cards, 90\%, 87\%, $73 \%$ and $74 \%$ ever used voice, SMS, data and USSD mCDRs, respectively. The number of SIM cards accessing the intervention declined marginally each month from $84 \%$ to $77 \%$ in the first seven months. In the eighth month, the number of SIM cards accessing the intervention abruptly declined to $67 \%$.

\section{Pattern of use of different intervention components}

The 307 SIM cards logged unto the Vodafone server 127 668 times altogether during the intervention period. Most of the time, the SIM cards were used to make phone calls (voice mCDRs 64\%), access the internet (data mCDRs 28\%), send SMS (5\%) and to access the USSD protocols $(2 \%)$ (table 3 ). This pattern of utilisation of the intervention components was observed when the Vodafone call $\log$ was analysed by district (except in the Asuogyman district), category of health facility (hospital, CHPS or HCs), location of the health facility (remote or non-remote) and the type of phone used (individual-use or shared-use phone) (see table 3). In the Asuogyaman district, the difference in pattern of utilisation of the intervention components was that the frequency of use of voice and data mCDRs were similar. Shared-use phones and phones designated to remotely located health facilities used the intervention less often compared with individual-use phones and non-remotely located health facilities.

When the data were analysed month by month for trends in utilisation of the intervention, it showed trends that were fairly steady in the first six months with some increase in use of voice calls around the third month and then an abrupt decline in all use around the sixth month. Figure 1 summarises these patterns of use of the different

Table 3 Pattern of mobile call detail record subtype use in the first eight months of intervention implementation

\begin{tabular}{|c|c|c|c|c|c|c|}
\hline \multirow[b]{2}{*}{ Variable } & \multirow{2}{*}{$\begin{array}{l}\text { Data } \\
\text { frequency }(\%)\end{array}$} & \multirow{2}{*}{$\begin{array}{l}\text { SMS } \\
\text { frequency (\%) }\end{array}$} & \multirow{2}{*}{$\begin{array}{l}\text { USSD } \\
\text { frequency (\%) }\end{array}$} & \multirow{2}{*}{$\begin{array}{l}\text { Voice } \\
\text { frequency (\%) }\end{array}$} & \multirow{2}{*}{$\begin{array}{l}\text { Total }^{\star} \\
\text { frequency (\%) }\end{array}$} & \multirow{2}{*}{$\begin{array}{l}\text { Total } \\
\text { frequency } \\
\text { per phone }\end{array}$} \\
\hline & & & & & & \\
\hline \multicolumn{7}{|l|}{ District (cluster) } \\
\hline Afram Plains North & $929(13.2)$ & $414(5.9)$ & $290(4.1)$ & $5431(76.9)$ & $7064(5.5)$ & 336.4 \\
\hline Asuogyaman & $5990(44.9)$ & $1056(7.9)$ & $372(2.8)$ & $5922(44.4)$ & $13340(10.5)$ & 460.0 \\
\hline Birim Central & $6191(33.1)$ & $890(4.8)$ & $549(2.9)$ & 11089 (59.2) & $18719(14.7)$ & 328.4 \\
\hline Denkyembour & $4028(24.5)$ & $1219(7.4)$ & $195(1.2)$ & $11003(66.9)$ & 16445 (12.9) & 401.1 \\
\hline Kwaebibiraem & 2487 (29.2) & $289(3.4)$ & $206(2.4)$ & $5524(64.9)$ & $8506(6.7)$ & 340.2 \\
\hline Kwahu West & $5036(21.9)$ & 744 (3.2) & $607(2.6)$ & 16622 (72.2) & 23009 (18.0) & 469.6 \\
\hline Lower Manya Krobo & $8096(29.2)$ & $1757(6.3)$ & 305 (1.1) & $17603(63.4)$ & $27761(21.7)$ & 514.1 \\
\hline West Akim & $3517(27.4)$ & $304(2.4)$ & $399(3.1)$ & $8604(67.1)$ & $12824(10.0)$ & 413.7 \\
\hline \multicolumn{7}{|l|}{ Type of health facility } \\
\hline Hospitals & $25144(28.4)$ & $4574(5.2)$ & $1841(2.1)$ & $58342(64.1)$ & 89901 (70.4) & 6915.5 \\
\hline Health centres & $6276(30.6)$ & $1003(4.9)$ & $568(2.8)$ & $12648(61.7)$ & $20495(16.1)$ & 706.7 \\
\hline CHPS and maternity homes & $4854(28.1)$ & $1096(6.4)$ & $514(3.0)$ & 10808 (62.6) & $17272(13.5)$ & 539.8 \\
\hline \multicolumn{7}{|l|}{ Type of phone } \\
\hline Individual-use & 30613 (29.0) & $4914(4.7)$ & 2311 (2.2) & $67573(64.1)$ & $105411(82.6)$ & 452.4 \\
\hline Shared-use & $5661(25.4)$ & $1759(7.9)$ & $612(2.6)$ & $14225(63.9)$ & 22257 (17.4) & 300.8 \\
\hline \multicolumn{7}{|l|}{ Demographic location } \\
\hline Non-remote & 31233 (29.0) & $5392(5.0)$ & $2306(2.1)$ & $68730(63.8)$ & $107661(84.3)$ & 2833.2 \\
\hline Remote & 5041 (25.2) & $1281(6.4)$ & 617 (3.1) & 13068 (65.3) & 20007 (15.7) & 555.8 \\
\hline
\end{tabular}

${ }^{*}$ Column percentages presented.

CHPS, Community Health Planning and Services; SMS, short messaging service; USSD, Unstructured Supplementary Service Data. 


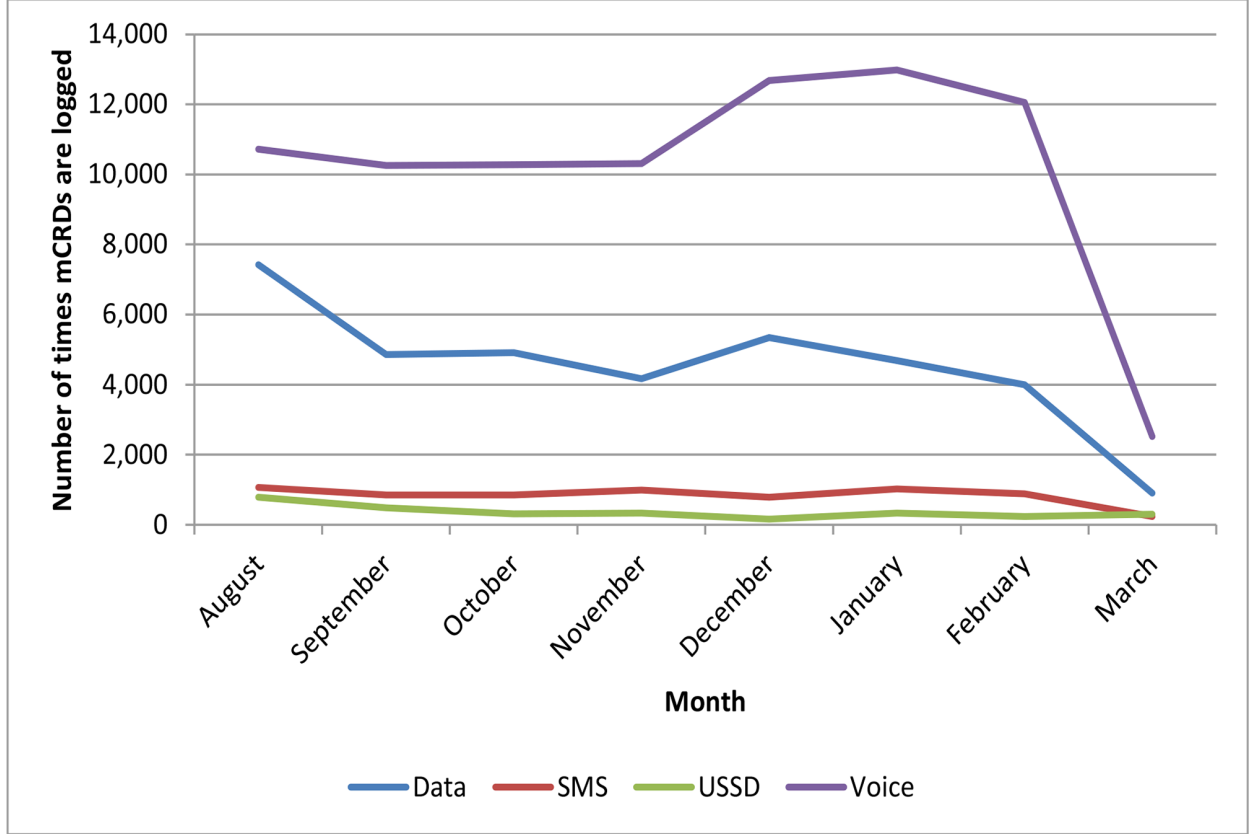

Figure 1 Trend in mobile call detail record use during the first eight months of intervention implementation. SMS, short messaging service; USSD, Unstructured Supplementary Service Data.

components of the intervention over time observed in all the eight intervention districts.

\section{Pattern of CUG communication}

Fifteen per cent of the 88471 records of voice and SMS communication with the projects phones were within the CUG. Nearly all CUG were voice mCDRs (97\%). At cluster level, majority of CUG communication was intracluster related ( $p$ value $<0.001$ ) (table 4$)$. The mean proportion of intra-cluster CUG communication was 0.96. Figure 2 maps the pattern of CUG communication between the clusters indicating inter-cluster communication, its frequency and the proximity or otherwise of clusters involved in the communication. Although CUG communication among the health facility types was varied ( $p$ value $<0.001$ ), there was little CUG communication across the different levels of health facilities. For example, there was little CUG communication between hospitals and HCs or between HCs and CHPS. With regard to health facility location, while non-remote areas communicated mostly (99\% of the time) within themselves ( $p$ value $<0.001$ ), remote areas communicated with other remote areas as often as they communicated with non-remote areas. Within the CUG, the pattern of shared-use and individual-use phone communication was not significantly different ( $p$ value $=0.065$ ). Details of SIM cards that used the CUG communication system in each cluster can be found in online supplementary appendix $1 \mathrm{~A}$ to 2.

\section{Characteristics of FGDs and KII participants}

Eight FGDs with a total of 54 respondents and 9 KIIs were conducted in total (table 5). Respondents were mainly midwives or community health nurses aged 26-76 years (mean age 38 years; $\mathrm{SD}=11$ years). Five respondents were male, the rest were female. Majority of respondents (31) held a certificate in midwifery or community health nursing as educational qualification. Most respondents maintained their key roles at work during the intervention period, although 12 of them changed work posts.

\section{Why were the patterns of use of intervention observed?}

The themes that emerged from our FGDs and KIIs analysis explaining the observed pattern of use of the intervention are summarised in figure 3 . The text that follows expands on each of these themes.

\section{Health worker factors}

Health worker demographics

Older midwives in the FGDs and KIIs reported that they made a lot of phone calls to their colleagues, friends and family. These older midwives often did not use data or send text messages because of unfamiliarity with the use of the internet or texting.

I knew but I am not conversant with the use of the internet, I was born before the computer. I wanted XXXXX to teach me, but she thought I was joking. Midwife, 50 years, HC (FGD 6)

... I did not know how to use it, that is why. I am now trying to learn it ... so my little my son is teaching me. Midwife, 55 years, $\mathrm{HC}$ (FGD 7 )

Younger midwifes were more curious and explored the use of data on social media particularly Facebook.

I was using it for Facebook ... because you will get free data to access Facebook ... we were enjoying small, small ... it is very fine because I liked the data. Midwife, 29 years, hospital (FGD 5) 
Table 4 Closed User Group voice and short messaging service (SMS) mobile call detail record communication using project mobile phones

\begin{tabular}{|c|c|c|c|c|c|c|}
\hline \multirow[b]{2}{*}{ Variable } & \multicolumn{3}{|l|}{ Voice } & \multicolumn{3}{|l|}{ SMS } \\
\hline & $\begin{array}{l}\text { Intra- } \\
\text { communication } \\
\text { frequency }(\%)\end{array}$ & $\begin{array}{l}\text { Inter- } \\
\text { communication } \\
\text { frequency }(\%)\end{array}$ & $\begin{array}{l}P \text { value for } \\
X^{2} \text { test }\end{array}$ & $\begin{array}{l}\text { Intra- } \\
\text { communication } \\
\text { frequency }(\%)\end{array}$ & $\begin{array}{l}\text { Inter- } \\
\text { communication } \\
\text { frequency }(\%)\end{array}$ & $\begin{array}{l}\text { P value for } \\
\text { Fisher's } \\
\text { exact test }\end{array}$ \\
\hline \multicolumn{7}{|l|}{ District (cluster) } \\
\hline Afram Plains North & 35 (100.0) & $0(0.0)$ & $<0.001$ & - & - & \\
\hline Asuogyaman & 437 (86.2) & 70 (13.8) & & $215(99.1)$ & $2(0.9)$ & $<0.001^{*}$ \\
\hline Birim Central & $636(96.1)$ & $26(3.9)$ & & $20(90.9)$ & $2(9.1)$ & \\
\hline Denkyembour & 3781 (95.2) & $192(4.8)$ & & $42(97.7)$ & $1(2.3)$ & \\
\hline Kwaebibiraem & $1306(92.8)$ & $102(7.2)$ & & $24(100.0)$ & $0(0.0)$ & \\
\hline Kwahu West & 1406 (99.3) & $10(0.7)$ & & $30(100.0)$ & $0(0.0)$ & \\
\hline Lower Manya Krobo & $3743(98.7)$ & $48(1.3)$ & & $73(90.1)$ & $8(7.9)$ & \\
\hline West Akim & $1248(99.8)$ & $2(0.2)$ & & 14 (93.3) & $1(6.7)$ & \\
\hline \multicolumn{7}{|l|}{ Type of health facility } \\
\hline Hospitals & 12460 (99.0) & $61(1.0)$ & $<0.001$ & $416(100.0)$ & $0(0.0)$ & $<0.001$ \\
\hline Health centres & $156(48.9)$ & $163(51.1)$ & & $1(14.3)$ & $6(85.7)$ & \\
\hline $\begin{array}{l}\text { Community Health Planning and } \\
\text { Services and maternity homes }\end{array}$ & $81(57.0)$ & $61(43.0)$ & & $9(100.0)$ & $0(0.0)$ & \\
\hline \multicolumn{7}{|l|}{ Type of phone } \\
\hline Shared-use & $13(2.8)$ & $452(97.2)$ & 0.065 & $0(0.00)$ & $4(100.0)$ & 1.00 \\
\hline Individual-use & $11993(95.4)$ & $584(4.6)$ & & $418(97.7)$ & $10(2.3)$ & \\
\hline \multicolumn{7}{|l|}{ Demographic location } \\
\hline Remote & $164(49.4)$ & $168(50.6)$ & $<0.001$ & $2(100.0)$ & $0(0.0)$ & $<0.001$ \\
\hline Non-remote & $101(0.8)$ & 12777 (99.2) & & 430 (100.0) & $0(0.0)$ & \\
\hline
\end{tabular}

${ }^{*} \chi^{2}$ test performed in this case.

\section{Personal and work-related needs of health worker}

Most FHWs made related phone calls to chat with friends within and outside the CUG or transmit information to their colleagues about work-related matters. For most FHWs, the phones bridged the communication gap at work and relieved the economic burden of having to use personal phones to make work-related phone calls especially in cases of non-functioning or non-existent work phones at the facility.

It was useful because when you do the call because this one you are not thinking about the credit on it so when you call you explain yourself to the person. Then the person can answer you clearly for you to understand what you are asking for.

For me it really helped because before then the calls I was making to my clients at times I felt I was incurring a lot of cost. So when it came I was not incurring any cost so I felt free and had no fear when calling any of my clients.

The FHWs explained that they used the project phones to follow-up defaulting antenatal clients, call doctors on duty to review cases, send reminders to clients to bring their neonates to the health facility for BCG vaccination, notify referral health facilities of pending referrals, clarify treatment given to referred patients and also make enquiries concerning action to be taken before referring a case to the referral centre as well as clarify handing over notes.

When non-functioning or non-existent work phones were replaced, fixed or supplied by health facility management (as was the case in a few health facilities in a few districts), then FHWs ceased to use the project phones for these calls.

\section{Perceived timeliness of the different intervention components to FHWs needs}

The voice mCDRs was perceived to be fast and timely by some FHWs while others thought that the USSD was faster. For those who preferred to make voice calls, they indicated that one was assured of contact with the person who was being called and hence information flow and rapid response to questions was guaranteed. All FHWs preferred voice calls to text messaging. They cited delays in text message delivery and not being sure the receiver of the text message would see the text at the time it was delivered as a preference for using voice mCDRs.

If you make a call, it will bell so even if she is asleep she will hear it and wake up but with the texting, if it is somewhere she wouldn't hear. Midwife 2, HC (FGD 3)

If you text that means the case is not so important, maybe you want to refer ... But if I know the case is a bleeding case 


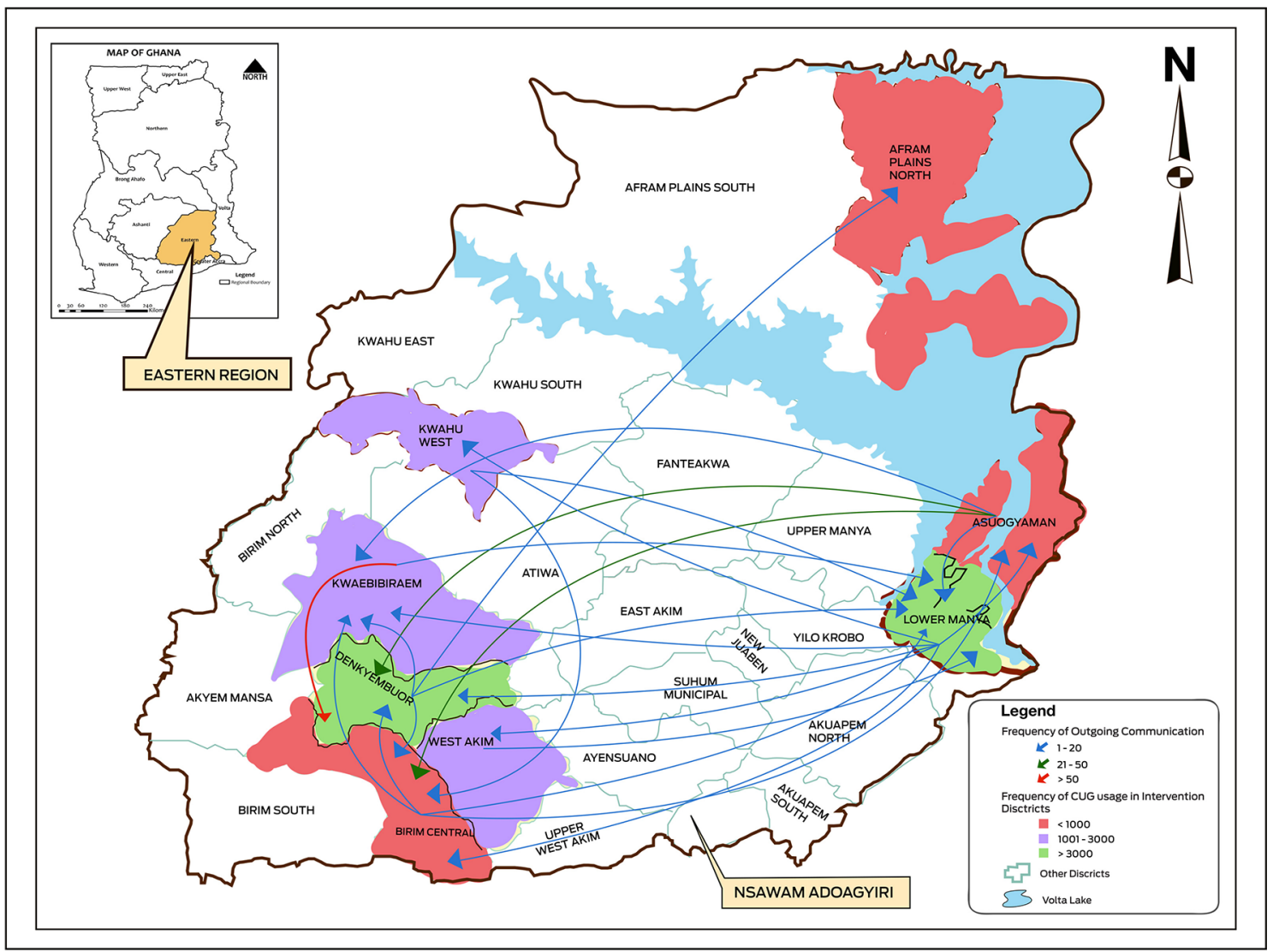

Figure 2 Mapping of pattern of Closed User Group (CUG) communication via phone calls and text messaging among clusters. The district colours indicate the frequency of CUG communication per cluster. The arrows show the direction of communication flow from one cluster to the other. The colour of the arrows indicate the frequency of inter-cluster communication with a given cluster. The pattern (frequency) of closed user group communication via phone calls and text messaging within and between the clusters as illustrated in figure 2 was significantly different ( $p$ value $<0.001)$.

or the condition is ... that is not the time to be pressing. I have to call to tell her. Midwife, HC (FGD 1)

FHWs who preferred to access the USSD indicated that the ease of use of the USSD platform, the simplified language of the protocols and the diversity of the USSD protocols was their motivation to use the USSD. These FHWs said they bypassed the inconvenience of having to access the protocols with gloved hands by learning the protocols beforehand.

It is not a long sentence. It is very short so you quickly read and you could just apply. 'Check her vital sign, $\mathrm{FH}$, how is it?' 'Do this-give antibiotics, after you are done refer her to the next level' so it was very quick ... It was simple with no big English. Midwife 2, hospital (FGD 3)

Table 5 Characteristics of respondent of focus group discussions and key informant interviews

\begin{tabular}{|c|c|c|c|c|c|c|c|c|c|c|}
\hline \multirow[b]{2}{*}{ District (cluster) } & \multicolumn{2}{|c|}{ Participants, $\mathbf{n}$} & \multirow[b]{2}{*}{$\begin{array}{l}\text { Age* }^{\star} \\
\text { (years) }\end{array}$} & \multicolumn{3}{|c|}{$\begin{array}{l}\text { Professional } \\
\text { training }\end{array}$} & \multicolumn{3}{|l|}{$\begin{array}{l}\text { Educational } \\
\text { qualification }\end{array}$} & \multirow{2}{*}{$\begin{array}{l}\text { Respondents } \\
\text { who received a } \\
\text { project phone, } \\
\text { n (\%) }\end{array}$} \\
\hline & FGDs & KII & & Midwife & $\mathrm{CHO}$ & Other† & Certificate‡ & $\begin{array}{l}\text { Diploma in } \\
\text { nursing }\end{array}$ & $\begin{array}{l}\text { Degree in } \\
\text { nursing }\end{array}$ & \\
\hline Afram Plains North & 3 & - & 30.0 & 1 & 2 & - & 1 & 2 & - & $1(33.3)$ \\
\hline Asuogyaman & 6 & 3 & 38.0 & 4 & 5 & - & 7 & - & 2 & $5(55.6)$ \\
\hline Birim Central & 6 & - & 29.0 & 4 & 2 & - & 4 & 2 & - & $5(83.3)$ \\
\hline Denkyembour & 6 & - & 31.5 & 4 & 2 & - & 3 & 2 & 1 & $6(100.0)$ \\
\hline Kwaebibiraem & 6 & - & 45.0 & 6 & - & - & 5 & 1 & - & $5(83.3)$ \\
\hline Kwahu West & 11 & - & 34.0 & 7 & 1 & 3 & 8 & 2 & 1 & 7 (63.6) \\
\hline Lower Manya Krobo & 5 & 4 & 48.0 & 7 & 1 & 1 & 8 & 1 & - & 8 (88.9) \\
\hline West Akim & 11 & 1 & 39.5 & 9 & 2 & 1 & 9 & 3 & - & $8(66.7)$ \\
\hline
\end{tabular}

One focus group discussion was conducted in each cluster; nine key informant interviews was conducted in three clusters.

*Median age reported.

†Includes three enrolled nurses, one ophthalmic nurse and medical assistant.

$\ddagger$ Certificate or post-basic qualification in midwifery or community health nursing.

$\mathrm{CHO}$, community health officer; FGD, focus group discussion; KII, key informant interview. 


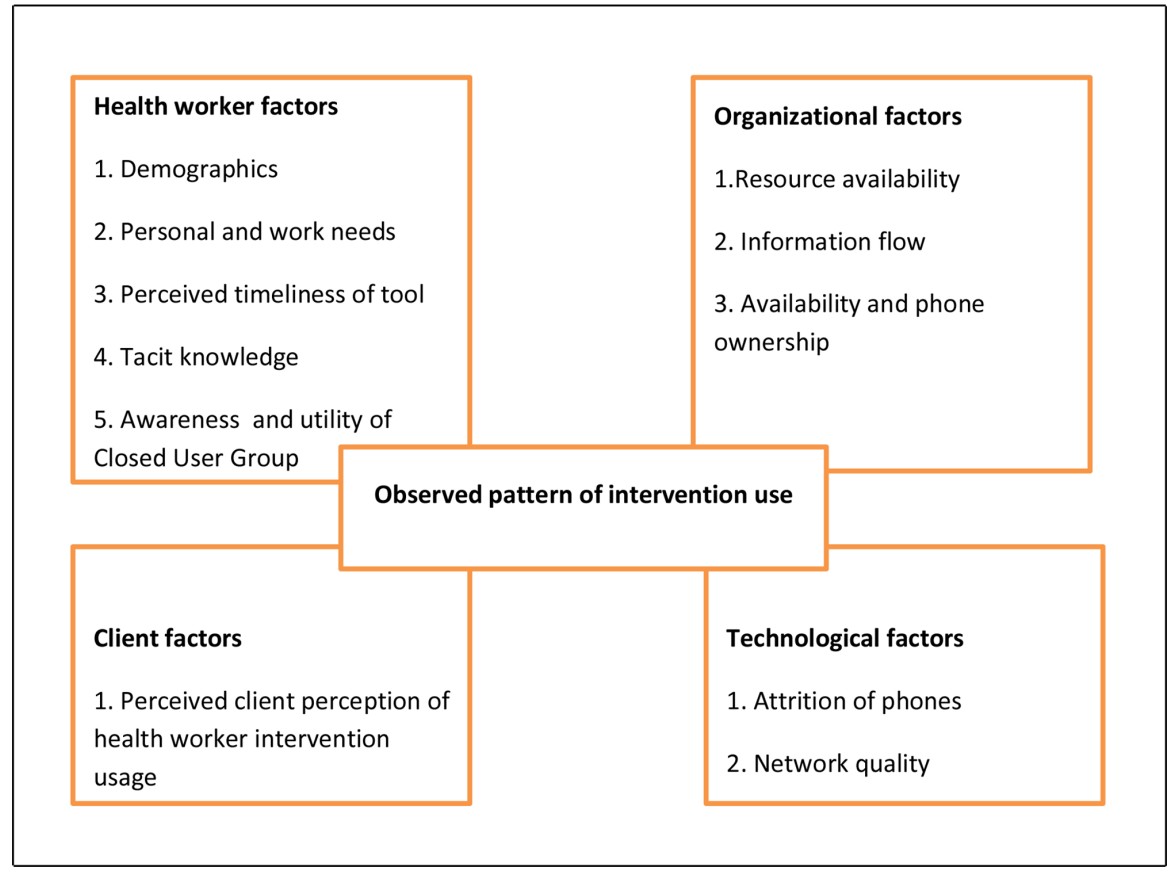

Figure 3 Factors explaining the observed pattern of mHealth intervention usage.

\section{Conversion of USSD protocols into 'mindlines'/tacit knowledge}

Initial use of the USSD component of the intervention was higher compared with its use in the subsequent months of intervention implementation. The USSD was used as a tool for revision and a reference to cross-check patient management. Over time, the FHWs memorised the protocols and did not have to refer to the USSD when confronted with cases. Some also wrote down the protocols and stopped consulting the phone.

It got to a point and it was like the protocols were stuck in my head. In our health center for instance, it was one-way cases. It's only once a while that we get something different. Like the PPH, asphyxia and others I have learnt it so it has stuck. As soon as it comes I know the protocol to use. Midwife, HC (FGD 6)

\section{CUG communication}

In two of the eight clusters, all FGD respondents knew about the CUG component of the intervention. In the remaining clusters, 50\% or more of FGDs respondents were unaware of this communication system. Most respondents who knew of the CUG thought there was a limit to the duration of the voice calls that could be made for free, which was not the case.

For us, when we use to call, we think we are using the credit on the phone, but we didn't know it was free. Midwife, hospital (FGD 8)

As phone numbers of the various users of the intervention were often not saved on the project phones, FHWs did not fully use the CUG communication system but called the personal phones of their colleagues using the project phones.

... sometimes you might be calling and she is not picking so you have to try her personal line because maybe the person is closer to her personal phone than project phone. So when you call and she is not picking then you call her personal line. Midwife, in HC (FGD 1)

In a few clusters, FHWs saved the project SIM numbers of their colleagues on their project phones.

... for those numbers, we gave them to our other colleagues who are at the health centers ... so we also stored their numbers and their names. When they want to refer a case, maybe if they want something then they call through the project ....Midwife 1, hospital (FGD 1)

\section{Organisational factors}

\section{Resource availability of health facilities}

In endowed hospitals where specialist obstetricians, paediatricians, doctors and senior midwives were readily available, FHWs found it more convenient to call their colleagues and doctors to review cases rather than check for protocols on their phone via USSD. These hospitals also had many protocols and regular morning meetings as a resource for continuing professional education.

Every Monday we used to have a presentation on the condition, so we are already abreast with whatever we are doing ... We had the protocols on the wall, when we are less busy we compared the ones we had on the phone to the ones we had at the wards and since we have studied it, when the condition comes, there is no need of us going to look at the protocols before we manage the condition. We manage it because we know the steps. Midwife, hospital (FGD 8)

\section{Information communication across the levels of the healthcare system}

At the health facilities, knowledge about the use of the phones was not readily shared with colleagues who were not present at the training; this included those who were newly posted to health facilities. Knowledge was also 
not transferred when trainees were posted out of their facilities even though they often left the project phones behind.

She went on pension ... she didn't even hand it over. The other midwife also came, and she was like "she did not hand over to me, so I will not touch it...

Also the person using the phone at the beginning was out of office and the one who succeeded him couldn't get that training so he couldn't apply like the first person.

Efficiency of communication from the project team to the various health facilities through the district health administration fell short with FHWs reporting to not have received follow-up information such as the list of all users of project phones and navigation menu for accessing the USSD sent to them. Likewise, reporting and handling of problems with the project phones (including reported cessation of monthly credit top-up) was ineffective as most respondents reported during the FGDs and KIIs that they had forgotten about the reporting system.

\section{Availability and ownership of shared-use phones}

Several FGD respondents (ranging from $0 \%$ to $70 \%$ per group) were unaware of a shared-use phone in their facilities and thus never assessed these phones. Many who were aware of these phones had personalised the phones and used them like individual-use phones. In other cases, the phone was kept under lock and key in a senior colleague's office cabinet.

... because she personalized it, when she is not around, you do not want to touch it unless the person is around then you ask her, if she gives you the permission, then you can touch it but if the person is not around you cannot touch it because you think it is her property.

... the facility phone was in the matron's office ... It is still in the box actually. The facility one was under lock and key ...

Where shared-use phones were readily available for use in health facilities, they were usually kept at a place known to all FHWs in the facility and someone was delegated to charge the phone. Readily available shared-use phones were usually used for community outreach purposes.

\section{Client perception of intervention}

In one cluster, respondents indicated that their preference for the voice mCDRs was because clients and their relatives might think that they were engaged in other activities like chatting on social media instead of attending to them if they saw them fiddling with their phones. However, when they made phone calls, client could hear that the conversation was about them (the client) and so clients felt that the FHWs were making an additional effort in their interest.

You see if you call and the person is standing there she won't get angry because she knows you are saying something about her relative. But when you are texting, the person will say the nurse is punching her phone and she is not minding us. If you are making the call then she will know it is either you have called a colleague or asking something from someone. Midwife 1, hospital (FGD 1)

But the moment she sees you pressing your phone ... they really hate it. Midwife 2, hospital (FGD 1)

This finding was however peculiar to only this cluster. Even within this cluster, one key informant did not think clients would have this perception if the midwife explained why she was taking a minute to fiddle with her phone.

\section{Technological factors Attrition of mobile phones}

Over time, respondents reported loss or theft of their individual-use phones usually in public transport vehicles. Most phone theft occurred at home. Project phones were also reported to have malfunctioned; commonly reported problems were charging issues, blank screens, freezing of phones, unresponsive keypads and non-functioning SIM cards. Some respondents also reported not being able to access the USSD platform despite obvious functioning of their project phones.

\section{Mobile network quality}

For most locations across the study site, the Vodafone network was good. Network quality, however, was poor in several rural areas; FHWs needed to stand or place the intervention phones at specific locations in order to be able to access network. For some, it was a 5 min drive on a motorbike to get good reception to access protocols. For others, being able to access network meant moving around the compound of the health facility in order to find a good spot.

Sometimes we have to move from the facility to a far place before we can access the network to do whatever we want to do. That is, it is the network which is affecting us.

\section{DISCUSSION}

\section{Pattern of use of different intervention components}

The high use of voice calls compared with all other components of the intervention suggests that in the study setting at least consideration should be given to mhealth interventions that allow voice calls. The preference for voice mCDRs for communication we observed is similar to findings from India, Bangladesh and Indonesia where mobile phone users preferred to use mobile phones for making calls than to text. ${ }^{33-35}$ Differential baseline knowledge and familiarity with use of mobile technologies among FHWs may have influenced the pattern of intervention usage observed. In this regard, FHWs most likely to use data (internet) in this study were mainly younger respondents.

Client perception of utilisation of mobile phones by HWs during consultation is documented in the literature. In India, patients were reported to have respect and confidence in health workers when they saw them accessing mHealth interventions during their consultation. ${ }^{213}$ Patients in Kenya, India and Indonesia believed that they will be provided the optimum care because 
FHWs accessed current information management choices using mHealth. ${ }^{22} 3738$ We identified a different reported client perception of health worker's utilisation of mHealth in this study. A small group of FHWs in our study were concerned that clients would think that they were engaged in other activities unrelated to their care if they accessed the USSD while attending to clients. The limitation of this observation to one cluster suggests that there were perhaps peculiar client-health worker dynamics in that cluster.

\section{Declined utilisation of mHealth intervention}

Over time, the use of all four mCDRs type declined. The attrition of mobile phones through theft, loss, malfunctioning phones and SIM cards problems could explain the decline in the number of SIM cards accessing the intervention and the decline in use of the intervention. Similar hardware challenges with electronic devices have been reported in other mHealth studies in LMICs and negatively impacted implementation of these interventions. ${ }^{21} 3940$ Feedback, refresher training and reminders for mHealth intervention users are known to sustain use of interventions ${ }^{2240-42}$; in this regard, monthly reminders to FHWs and on-site training performed during routine supervisory visits by the project team during intervention implementation were good. However, the absence of a feedback mechanism to FHWs concerning how the intervention was being used could have negatively impacted its sustained use.

In low-resource settings such as the study setting, it is not uncommon that an essential tool such as a work phone may be absent or non-functional because of challenges with maintaining these phones due to the high recurrent cost of phone calls and replacement of malfunctioning phones. Health workers in these settings often resort to using their personal phones and funds, albeit reluctantly, for work-related matters. ${ }^{22}$ It is therefore not surprising that FHWs in this study readily used the project phones to make phone calls related to work- calling their clients, other colleagues, doctors and referral points as noted in other studies. ${ }^{22}{ }^{43}$ The decline in voice mCDRs, in this study was partly related to the replacement of malfunctioning work phones removing the need to use project phones for phone calls. When FHWs did not receive the monthly top-up on their phones to assure free calls outside the CUG, it was a demotivation to use the intervention. This demotivation was further reinforced by FHWs lack of knowledge that CUG communication was free and unlimited.

This study shows that the USSD protocols become part of FHWs' tacit knowledge very quickly. Health workers often use tacit knowledge in clinical decision-making. ${ }^{18}$ The rapid internalisation of the protocols not only explains the decline in accessing the USSD but also suggests that the protocols were useful, simple and easy to memorise. Poor network quality may have reinforced the need to commit the protocols into memory. Previous studies have found that network quality influenced the uptake and confidence in mHealth interventions studies. $^{622} 2334$

\section{Pattern of CUG communication}

Most $(85 \%)$ communication with other SIM cards using the project phones was non-CUG related. Contact made with clients, friends and family explains the high proportion of non-CUG communication observed. The majority of FHWs did not save the project phone numbers of their colleagues on their project phones, as such, in communicating with colleagues who were intervention users, they keyed the personal numbers of their colleagues on the project phones. This further pushed up the proportion of non-CUG voice mCDRs. Health workers may have not saved the phone numbers of their colleagues on the project phone because they knew they could access it on their personal phones. We report low awareness of the CUG communication system in this study. The limited knowledge of the CUG is demonstrated by the low number of SIM cards involved in the CUG communication in the clusters (online supplementary appendix 1 and 2). Perhaps knowledge of the CUG and a mechanism that allowed FHWs to easily search and find their colleagues within the CUG would have been a motivation to use the CUG more often. Low awareness of mHealth interventions and their functions has been documented as a barrier to mHealth intervention use. ${ }^{44}$ Reminders and reward schemes (tangible or intangible) can motivate users to use mHealth applications. ${ }^{42} 4445$ The reasons for using the voice and SMS intervention components could be situated in the mapping of the CUG communication. For example, one could anticipate the proximity or otherwise of work-related use (eg, support and referrals) and non-work-related use of the phones between the various clusters from the CUG map and the possible consequence of this pattern of CUG communication on outcomes estimated in future analysis of the impact of the intervention on beneficiary outcomes.

\section{Pattern of utilisation of project phones according to sharing status of phone and health facility location}

Our observed higher use of individual-use phones compared with shared-use phones as well as the tendency to personalise or lock up shared phones for safety suggests that mHealth intervention designs delivered through individualised rather than shared device may be more effective. mHealth interventions that allow FHWs access with their own phones could also assure universal access for users in this regard. The low intervention usage in remotely located health facilities correlates with the sharing status of phones; most remotely located health facilities lacked midwives and so received shared facility phones. Poor network quality also negatively influenced the use of phones in remote areas.

\section{Limitations}

There are some limitations to this study. First, the analysis of the Vodafone call $\log$ is limited to the first eight 
months of an 18-month intervention. It would be useful to analyse data for the entire 18 months; however, this was impossible due to constraints of data retrieval from Vodafone Ghana at the time of analysis. Our quantitative data analysis cannot determine whether the content of phone calls made were work-related or not. Neither are we able to ascertain the degree to which phone numbers called outside the CUG were indeed numbers of other health workers. While the use of project phones for non-related calls may be undesirable from management's view, one may argue that unrestricted use of phones could motivate target groups of mHealth interventions to use the interventions. Third, facility heads selected respondents for this study; the criteria for respondent selection are unknown and may have influenced results. Lastly, in-depth understanding of how and why the phones were used in context required a qualitative study. We have obtained this understanding. However, we cannot assume that our results are transferable beyond the study setting. Despite these limitations, this study provides valuable information regarding preference and usability of a multifaceted mHealth intervention among FHWs in a low-resource setting. The findings from this study can inform recommendations in the design and scale up of mHealth interventions in low-resource settings.

\section{CONCLUSION}

How and why m-Health interventions are used (or not) goes beyond the technology itself and are influenced by individual and context-specific factors. We identified factors that influenced the uptake of a multifaceted mHealth intervention for clinical decision-making. Knowledge of these factors can guide the design of mHealth interventions whose components are similar to the individual components of this mHealth intervention. This study further reinforces the need for usability studies to optimise successful implementation of affordable mHealth solutions in LMICs.

\section{Author affiliations \\ ${ }^{1}$ School of Public Health, University of Ghana, Accra, Ghana \\ ${ }^{2}$ Julius Global Health, Julius Center for Health Sciences and Primary Care, University Medical Center, Utrecht University, Utrecht, The Netherlands ${ }^{3}$ Division of Epidemiology and Biostatistics, School of Public Health, Faculty of Health Sciences, University of the Witwatersrand, Johannesburg, South Africa ${ }^{4}$ University of Health and Allied Sciences, Ho, Ghana ${ }^{5}$ Ghana Health Service, Research and Development Division, Dodowa Research Centre, Accra, Ghana}

Acknowledgements The authors thank the Ghana Health Service Research and Development Division, the Eastern Region Health Directorate, members of the various district health management teams in the Eastern Region and the School of Public Health, University of Ghana for their support in the conduct of this study. They also thank Richard Kwofie, Theophilus Botchway and Ebenezer Amankwah of Vodafone Ghana for their support in extracting the call log data and Emmanuel Amankwah of Dela \& Amankwah Limited for his support in designing the map used in this paper.

Contributors HBA, KKG, EA, LY and IAA designed and performed the study and analysed the data. HBA drafted the manuscript and KKG, EA and IAA reviewed the manuscript. DEG and LY provided critical comments on the review of the manuscript. All authors have read and approved the final manuscript.
Funding The Netherlands Foundation for Scientific Research, Global Health Policy and Health Systems Research Program (grant number: 07.45.102.00) and the Julius Center, University Medical Centre, Utrecht, Netherlands, provided financial support. HBA has been supported by a Global Health Scholarship Grant from the University Medical Center Utrecht.

Competing interests None declared.

Patient consent for publication Not required.

Ethics approval Ethics approval for this study was obtained from the Ghana Health Service Ethics Review committee (approval number: GHS-ERC: 04/09/16) before this study commenced.

Provenance and peer review Not commissioned; externally peer reviewed.

Data sharing statement Data are available on request by emailing the corresponding author at ansomaame@hotmail.com.

Open access This is an open access article distributed in accordance with the Creative Commons Attribution Non Commercial (CC BY-NC 4.0) license, which permits others to distribute, remix, adapt, build upon this work non-commercially, and license their derivative works on different terms, provided the original work is properly cited, appropriate credit is given, any changes made indicated, and the use is non-commercial. See: http://creativecommons.org/licenses/by-nc/4.0/.

\section{REFERENCES}

1. Labrique $A B$, Vasudevan L, Kochi E, et al. mHealth innovations as health system strengthening tools: 12 common applications and a visual framework. Global Health: Science and Practice 2013;1:160-71.

2. Agarwal S, Perry HB, Long LA, et al. Evidence on feasibility and effective use of mHealth strategies by frontline health workers in developing countries: systematic review. Trop Med Int Health 2015;20:1003-14.

3. Sondaal SF, Browne JL, Amoakoh-Coleman M, et al. Assessing the effect of mHealth interventions in improving maternal and neonatal care in low- and middle-income countries: a systematic review. PLoS One 2016;11:e0154664.

4. Hall CS, Fottrell E, Wilkinson S, et al. Assessing the impact of mHealth interventions in low- and middle-income countries--what has been shown to work? Glob Health Action 2014;7:25606-12.

5. Mehl GL, Tamrat T, Bhardwaj S, et al. Digital health vision: could MomConnect provide a pragmatic starting point for achieving universal health coverage in South Africa and elsewhere? BMJ Glob Health 2018;3.e000626.

6. Lemay NV, Sullivan T, Jumbe B, et al. Reaching remote health workers in Malawi: baseline assessment of a pilot mHealth intervention. J Health Commun 2012;17 Suppl 1:105-17.

7. Oyeyemi SO, Wynn R. The use of cell phones and radio communication systems to reduce delays in getting help for pregnant women in low- and middle-income countries: a scoping review. Glob Health Action 2015;8:28887.

8. Betjeman TJ, Soghoian SE, Foran MP. mHealth in Sub-Saharan Africa. Int J Telemed Appl 2013;7.

9. Abbott PA, Foster J, Marin HF, et al. Complexity and the science of implementation in health IT-knowledge gaps and future visions. Int $J$ Med Inform 2014;83:e12-22.

10. Johnson CM, Johnson TR, Zhang J. A user-centered framework for redesigning health care interfaces. J Biomed Inform 2005;38:75-87.

11. Zhang S, Wu Q, van Velthoven $\mathrm{MH}$, et al. Smartphone versus penand-paper data collection of infant feeding practices in rural China. $J$ Med Internet Res 2012;14:e119.

12. Adepoju IO, Albersen BJ, De BrouwereV, et al. mHealth for clinical decision-making in sub-Saharan Africa: a scoping review. JMIR Mhealth Uhealth 2017;5:e38.

13. Blank A, Prytherch H, Kaltschmidt J, et al. "Quality of prenatal and maternal care: bridging the know-do gap" (QUALMAT study): an electronic clinical decision support system for rural Sub-Saharan Africa. BMC Med Inform Decis Mak 2013;13:44.

14. McNabb M, Chukwu E, Ojo O, et al. Assessment of the quality of antenatal care services provided by health workers using a mobile phone decision support application in northern Nigeria: a pre/postintervention study. PLoS One 2015;10:e0123940.

15. Horner V, Rautenbach P, Mbananga N, et al. An e-health decision support system for improving compliance of health workers to the maternity care protocols in South Africa. Appl Clin Inform 2013;4:25-36. 
16. Bank TW, 2016. Maternal mortality ratio (modeled estimate, per 100,000 live births) | Data | Table. Available from: http://data. worldbank.org/indicator/SH.STA.MMRT [Accessed 27 Jan 2016].

17. Ghana Statistical Service (GSS), Ghana Health Service (GHS) and I, 2018. Ghana maternal health survey 2017 key indicators. Available from: http://www.statsghana.gov.gh/docfiles/PR95.pdf [Accessed 5 Jun 2018].

18. Oduro-Mensah E, Kwamie A, Antwi E, et al. Care decision making of frontline providers of maternal and newborn health services in the greater Accra region of Ghana. PLoS One 2013;8:e55610.

19. Accelerate Project, 2015. Available from: http://www.acceleratemdg org/ [Accessed 2 Jul 2018]

20. Amoakoh HB, Klipstein-Grobusch K, Amoakoh-Coleman M, et al. The effect of a clinical decision-making mHealth support system on maternal and neonatal mortality and morbidity in Ghana: study protocol for a cluster randomized controlled trial. Trials 2017;18:157.

21. Praveen D, Patel A, Raghu A, et al. SMARTHealth India: development and field evaluation of a mobile clinical decision support system for cardiovascular diseases in rural India. JMIR Mhealth Uhealth 2014;2:e54.

22. Chib A, Lwin MO, Ang J, et al. Midwives and mobiles: using ICTs to improve healthcare in Aceh Besar, Indonesia1. Asian J Commun 2008;18:348-64.

23. Vedanthan R, Blank E, Tuikong N, et al. Usability and feasibility of a tablet-based decision-support and integrated record-keeping (DESIRE) tool in the nurse management of hypertension in rura western Kenya. Int J Med Inform 2015;84:207-19.

24. The World Bank Group, 2018. World bank open data. Available from https://data. worldbank.org/country/ghana?view=chart [Accessed 21 Jun 2018]

25. World Health Organization, 2015. Ghana:WHO statistical profile. Geneva, Switzerland. Available from: http://www.who.int/gho/ countries/gha.pdf?ua=1 [Accessed 21 Jun 2018].

26. WHO, UNICEF, UNFPA WBG, and the United Nations Population Division, 2015. Trends in maternal mortality: 1990 to 2015 estimates by WHO, UNICEF, UNFPA, world bank group and the united nations population division. Geneva, Switzerland. Available from: https://data.unicef.org/wp-content/uploads/2015/12/MMR executive summary final_mid-res 243.pdf [Accessed $31 \mathrm{Jul}$ 2018].

27. Ghana Statistical Service (GSS), Ghana Health Service (GHS) and II, 2015. Ghana demographic and health survey 2014. Accra, Ghana. Available from: https://dhsprogram.com/pubs/pdf/fr307/fr307.pdf [Accessed 21 Jun 2018].

28. Ghana Statistical Service, 2012. 2010 Population \& housing census summary report of final results. Accra, Ghana: : Ghana Statistical Service. Available from: http://www.statsghana.gov.gh/docfiles/ 2010phc/Census2010 Summary report of final results.pdf

29. Ghana Demographic and Health Survey 2014,2015 . Available from: http://www.statsghana.gov.gh/docfiles/publications/Ghana DHS 2014 - KIR - 6 April 2015 [Accessed 9 Mar 2016].

30. Corporation M. Microsoft Ecxel 2010.
31. Guest G, Namey E, McKenna K. How many focus groups are enough? Building an evidence base for nonprobability sample sizes. Field methods 2017;29:3-22.

32. StataCorp, 2013. Stata statistical software: release 13. college station, TX: stataCorp LP. Available from: http://www.stata.com/ support/faqs/resources/citing-software-documentation-faqs [Accessed 28 Jan 2016]

33. DeSouza SI, Rashmi MR, Vasanthi AP, et al. Mobile phones: the next step towards healthcare delivery in rural India? PLoS One 2014:9:e104895.

34. Jain N, Singh H, Koolwal GD, et al. Opportunities and barriers in service delivery through mobile phones (mHealth) for severe mental illnesses in Rajasthan, India: a multi-site study. Asian J Psychiatr 2015;14:31-5.

35. Khatun F, Heywood AE, Ray PK, et al. Determinants of readiness to adopt mHealth in a rural community of Bangladesh. Int $J$ Med Inform 2015;84:847-56.

36. Gautham M, lyengar MS, Johnson CW. Mobile phone-based clinical guidance for rural health providers in India. Health Informatics $J$ 2015;21:253-66.

37. Jones CO, Wasunna B, Sudoi R, et al. "Even if you know everything you can forget": health worker perceptions of mobile phone textmessaging to improve malaria case-management in Kenya. PLoS One 2012; 7:e38636

38. Mwendwa P. Assessing the fit of RapidSMS for maternal and newborn health: perspectives of community health workers in rural Rwanda. Dev Pract 2016;26:38-51.

39. Medhanyie AA, Little A, Yebyo $\mathrm{H}$, et al. Health workers' experiences, barriers, preferences and motivating factors in using mHealth forms in Ethiopia. Hum Resour Health 2015;13:2

40. Ngabo F, Nguimfack J, Nwaigwe F, et al. Designing and Implementing an Innovative SMS-based alert system (RapidSMS$\mathrm{MCH}$ ) to monitor pregnancy and reduce maternal and child deaths in Rwanda. Pan Afr Med J 2012;13:31.

41. Haberer JE, Kiwanuka J, Nansera D, et al. Challenges in using mobile phones for collection of antiretroviral therapy adherence data in a resource-limited setting. AIDS Behav 2010;14:1294-301.

42. Mwendwa P. What encourages community health workers to use mobile technologies for health interventions? Emerging lessons from rural Rwanda. Development Policy Review 2018;36:111-29.

43. Ran Van Der Wal K, 2018. Acceptability and use of mHealth tools by auxiliary midwives in Myanmar: a qualitative study. Available from: https://papyrus.bib.umontreal.ca/xmlui/bitstream/handle/1866/ 18620/van_der_Wal_KyongRan_2016_memoire.pdf?sequence=4 [Accessed 17 May 2018].

44. Peng W, Kanthawala S, Yuan S, et al. A qualitative study of user perceptions of mobile health apps. BMC Public Health 2016;16:1-11.

45. Zurovac D, Sudoi RK, Akhwale WS, et al. The effect of mobile phone text-message reminders on Kenyan health workers' adherence to malaria treatment guidelines: a cluster randomised trial. Lancet 2011;378:795-803. 